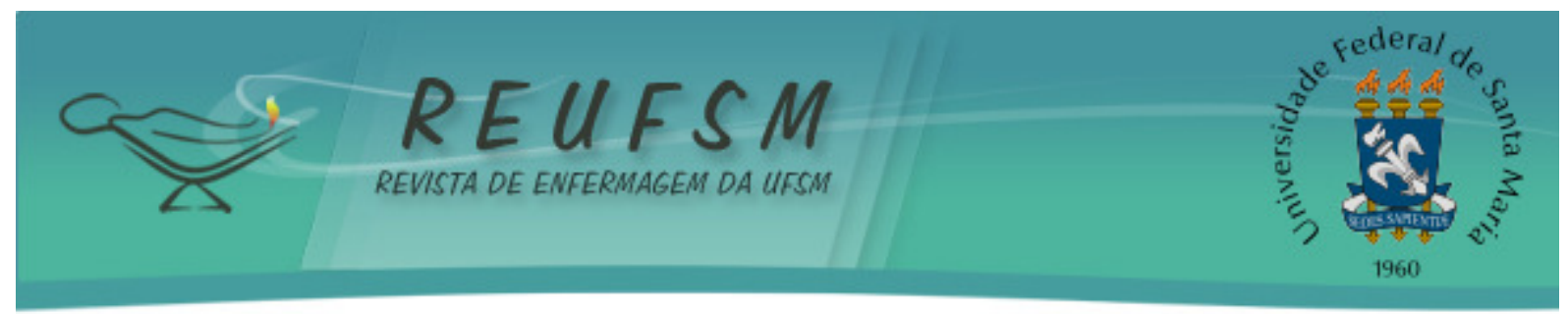

ARTIGO ORIGINAL

\title{
QUANTIFICAÇÃO DE RISCO PARA DOENÇAS CARDIOVASCULARES EM USUÁRIOS DE UMA ESTRATÉGIA DE SAÚDE DA FAMÍLIA
}

\section{RISK MESUREMENT OF CARDIOVASCULAR DISEASE IN FAMILY HEALTH STRATEGY USERS \\ MEDICIÓN DEL RIESGO DE ENFERMEDADES CARDIOVASCULARES EN USUARIOS DE UNA ESTRATEGIA DE SALUD DE LA FAMILIA}

\author{
Marcelo Machado Sassi ${ }^{1}$ \\ Gianfábio Pimentel Franco ${ }^{2}$ \\ Sidnei Petroni ${ }^{3}$ \\ Débora Dalegrave ${ }^{4}$ \\ Gabriela de Mattos Nogueira ${ }^{5}$
}

Doi: $10.5902 / 2179769213396$

RESUMO: Objetivo: quantificar o risco para a ocorrência de doenças cardiovasculares em usuários de uma Estratégia da Saúde da Família. Método: estudo quantitativo, não experimental, descritivo e com recorte de tempo transversal. Para quantificação do risco utilizou-se o software Framingham General Cardiovascular Disease. Resultados: foram entrevistados 52 sujeitos, destes 6 apresentaram grau de risco adequado para a idade, 27 apresentaram grau de risco elevado para a idade e 19 grau de alto risco para a idade. Conclusão: a quantificação de risco possibilita visualizar um panorama a respeito de determinada situação, e assim, traçar planos de ações de intervenção.

Descritores: Fatores de risco; Enfermagem; Atenção primária à saúde; Doenças cardiovasculares.

ABSTRACT: Aim: To quantify the risk on the occurrence of cardiovascular diseases in Family Health Strategy users. Method: quantitative, non-experimental, descriptive and cross-cut time study. For quantification of risk the Framingham General Cardiovascular Disease software was used. Results: 52 subjects were interviewed, 6 of them presented a degree of risk appropriated for their age, 27 had a high level risk for their age and 19 high-risk for their age. Conclusion: risk quantification enables the view on a situation, and thus to draw action plans for intervention.

Descriptors: Risk factors; Nursing; Primary health care; Cardiovascular diseases.

RESUMEN: Objetivo: Evaluar el riesgo para la ocurrencia de enfermedades cardiovasculares en los usuarios de una Estrategia de Salud de la Familia. Método: cuantitativo, no experimental, descriptivo y estudio transversal del tiempo. Para la

\footnotetext{
${ }^{1}$ Enfermeiro graduado pela Universidade Federal de Santa MarialPalmeira das Missões. Residente em Urgência e Emergência no Hospital Universitário da Universidade Federal de Santa Catarina - UFSC. Brasil. E-mail: sassimarcelomachado@yahoo.com.br

2 Enfermeiro, Doutor em Ciências pela Universidade Federal de São Paulo - UNIFESP, Professor Adjunto IV do Curso de Enfermagem da Universidade Federal de Santa MarialCentro de Educação Superior Norte do Estado do RS (UFSMICESNORS). Palmeira das Missões, RS. Brasil. E-mail: gianfabiofranco@yahoo.com.br

${ }^{3}$ Biólogo, Doutor em Anatomia pela Universidade Estadual Paulista “Júlio de Mesquita Filho" - UNESP, Professor Adjunto III do Curso de Enfermagem da Universidade Federal de Santa MarialCentro de Educação Superior Norte do Estado do RS (UFSMICESNORS). Palmeira das Missões, RS. Brasil. E-mail: sidneipetroni@yahoo.com.br

4 Enfermeira do Hospital Divina ProvidêncialFrederico Westphalen\RS, Mestre em Enfermagem pela Universidade Federal do Rio Grande do Sul - UFRGS. Brasil. E-mail: debora_dalegrave@yahoo.com.br

5 Enfermeira do Hospital de Caridade de Palmeira das Missões, Especialista em Terapia Intensiva pela Universidade Regional do Noroeste do Estado do Rio Grande do Sul - UNIJUí. Brasil. E-mail: gaby_mnog@hotmail.com
} 


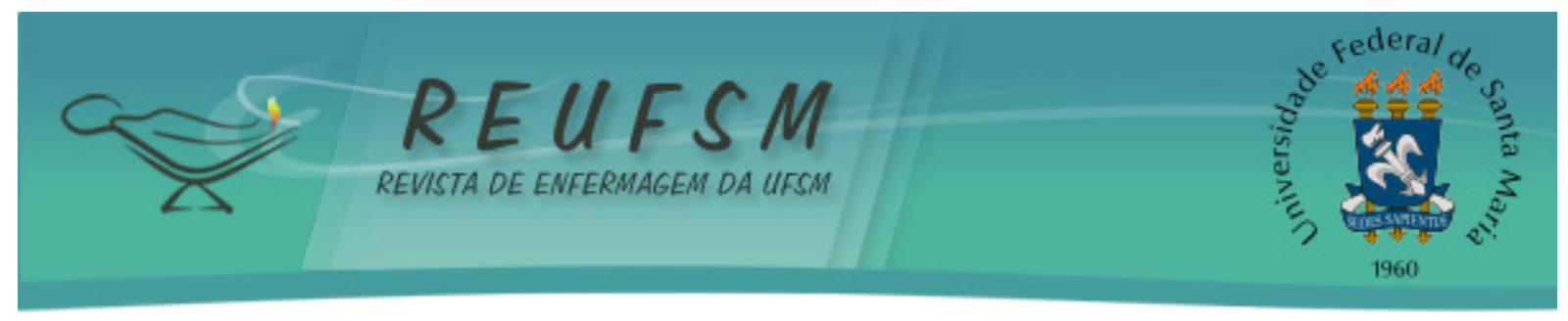

medición del riesgo fue utilizado el software Framingham General Cardiovascular Disease. Resultados: 52 sujetos fueron entrevistados, de estos, 6 presentaron nivel de riesgo apropiado para la edad, 27 tenían alto nivel de riesgo para la edad y 19 alto riesgo para la edad. Conclusiones: la cuantificación del riesgo permite una vista panorámica sobre una determinada situación, y así, dibujar planes de acción para la intervención.

Descriptores: Factores de riesgo; Enfermería; Atención primaria de salud; Enfermedades cardiovasculares.

\section{INTRODUÇÃO}

As doenças cardiovasculares constituem atualmente a principal causa de morbidade e mortalidade no mundo, sendo este um dos principais problemas de saúde pública. ${ }^{1}$ No Brasil, segundo dados do Departamento de Informática do Sistema Único de Saúde (DATASUS) do ano de 2012 e de acordo com a Classificação Internacional de Doenças 10 (CID-10), em todo o país, 333.295 pessoas morreram em decorrência de doenças do aparelho circulatório, sendo esta a primeira causa de morte no país, seguida pelas neoplasias com 191.157 mortes, e pelas causas externas de morbidade e mortalidade com 152.013 mortes.

As principais doenças cardiovasculares, agrupadas no CID-10 e notificadas como causas de mortes no Brasil no referido ano, foram as doenças isquêmicas do coração, com 104.397 óbitos e as doenças cerebrovasculares com 100.194 mortes. O estado do Rio Grande do Sul apresentou a segunda maior taxa de mortalidade do país, com 209 óbitos por 100.000 habitantes, superado somente pelo estado do Rio de Janeiro, com 229 mortes por 100.000 habitantes. No município de Palmeira das Missões, foco deste estudo, no ano de 2012 ocorreram 64 mortes por doenças do aparelho circulatório, correspondendo a $33,0 \%$ do total de óbitos da cidade.

As doenças cardiovasculares também foram a principal causa de internações hospitalares, somente no ano de 2010 foram realizadas 1.153 .213 internações em todo país causadas pelas referidas doenças, com uma media de 5,1 dias de permanência hospitalar e uma taxa de mortalidade de 5,4\%. Como decorrência, o Sistema Único de Saúde (SUS), demandou a quantia de $\mathrm{R} \$ 2$ 2.094.586.170,18 para cobrir as referidas despesas hospitalares, tal gasto correspondeu a cerca de $20,0 \%$ do orçamento total do SUS gasto no ano de 2010 com todas as internações. Ainda, no município de Palmeira das Missões/RS foram demandados R\$ 232.314,50 com internações hospitalares devido a doenças cardiovasculares. ${ }^{2}$

Os avanços recentes em nível tecnológico e científico na área da saúde melhoraram o prognóstico das doenças cardiovasculares, entretanto é evidente por meio do elevado número de morbidade e mortalidade causado por estes agravos, que o controle de seus fatores de risco ainda é insuficiente. ${ }^{3}$ Além disso, no Brasil, persiste a carência de estudos de prevalência destas doenças e dos seus fatores de risco modificáveis no contexto populacional. ${ }^{4}$

A ocorrência de uma doença cardiovascular está associada a fatores de riscos, que podem ser definidos como um aspecto do comportamento pessoal ou estilo de vida, uma exposição ambiental ou uma característica inata ou herdada, que com base na evidencia epidemiológica está associada a ocorrência da doença. ${ }^{5}$ Os resultados obtidos no Framingham Heart Study apresentaram, pela primeira vez, a confirmação científica de alguns fatores de risco de maior incidência para o acometimento de doenças cardiovasculares, entre eles, o tabagismo, a hipertensão arterial sistêmica (HAS), as dislipidemias e o diabetes mellitus (DM). ${ }^{6}$ 


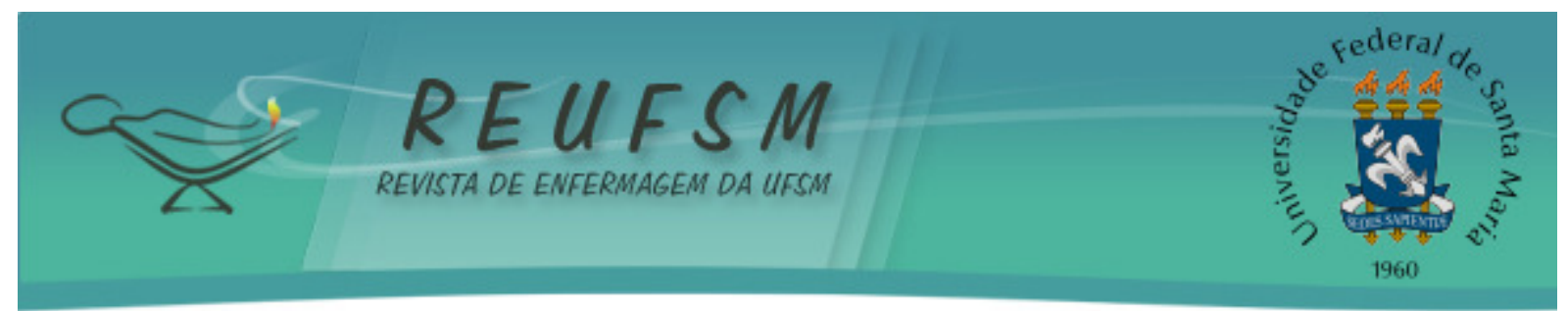

Já o sedentarismo, a obesidade central e periférica foram posteriormente acrescentados como fatores de risco importantes para o desenvolvimento de doenças cardiovasculares. ${ }^{7}$ Entretanto, entre os maiores desafios no combate a redução de mortes por doenças cardiovasculares estão o controle do sobrepeso ou da obesidade e do sedentarismo. ${ }^{8}$ A redução destes fatores de risco são indispensáveis para a prevenção de doenças cardiovasculares. As estimativas apontam que $22,0 \%$ das doenças isquêmicas do coração tenham como fator responsável o sedentarismo, ainda que este seja dependente de outros fatores associados. ${ }^{9}$

Dados relativos a prevalência de fatores de risco para doenças cardiovasculares e outras doenças crônicas apontam que, em nível nacional, a população brasileira, no ano de 2010, apresentou-se como portadora de DM em 9,9\%. A região sul possui a segunda maior prevalência, com cerca de $10,5 \%$, superado somente pela região sudeste, com $10,7 \%$.

A HAS é o fator de risco mais freqüente para as doenças cardiovasculares, se apresentando no país em cerca de $23,3 \%$ da população, cabe a região sudeste a maior prevalência, com $25,2 \%$, seguida pela região sul com $23,8 \%$. Em relação aos tabagistas, os dados apontam que $15,1 \%$ da população brasileira são fumantes, estando a região sul com a mais alta prevalência entre todas as regiões do país com $18,1 \%$, seguida da região sudeste com $17,2 \%$.

Com relação ao sedentarismo, é citado como outro fator de risco preocupante, pois apenas $14,9 \%$ da população brasileira praticam alguma atividade física de forma suficiente, a região sudeste e a região sul apresentam os menores índices com 13,8\% e $15,1 \%$, respectivamente. Estes aspectos indicam a elevada porcentagem da população no Brasil com excesso de peso, 48,5\%, e com obesidade $14,5 \%$, onde a região sul apresenta as maiores prevalências com $52,3 \%$ do total de sua população com excesso de peso e $25,7 \%$ com obesidade. O consumo abusivo de álcool é outro fator prevalente em $18,0 \%$ da população brasileira, a região nordeste com $21,2 \%$ e a região norte com $19,0 \%$ apresentam os maiores índices dentre as demais regiões do país. ${ }^{1}$

Ao analisar a etiologia de um indivíduo e sua relação com crenças pessoais podese verificar que essas podem estar relacionadas com a percepção subjetiva do sujeito para adquirir determinada doença ou em adotar certas medidas terapêuticas. ${ }^{10}$ Percebe-se que a importância em identificar e classificar os fatores de risco não pode ser dissociada do contexto social no qual as pessoas estão inseridas e de seu estilo de vida. Valores culturais e crenças, situações socioeconômicas e aspectos cognitivos necessitam serem considerados, pois podem implicar diretamente no processo de adoecer e cuidar de si. ${ }^{11}$ Assim, a Estratégia de Saúde da Família (ESF) detém grande potencial para atuar na redução dos fatores de risco das doenças cardiovasculares, através do estimulo a mudanças de hábitos de vida, individuais e/ou coletivos.

Considerando o exposto, este estudo teve por objetivo quantificar o risco para a ocorrência de doenças cardiovasculares em usuários de uma ESF do município de Palmeira das Missões/RS.

\section{MÉTODO}

Trata-se de um estudo quantitativo, com delineamento não experimental, de caráter descritivo e recorte de tempo transversal. ${ }^{12}$ Os dados foram coletados por meio de um questionário estruturado pelos autores com as variáveis de idade, sexo, diabetes mellitus, tabagismo, índice de massa corporal e o uso de tratamento medicamentoso para HAS.

A escolha dos sujeitos da amostra se deu por conveniência, de acordo com atendimento de livre demanda e de agendamentos em uma ESF de Palmeira das 


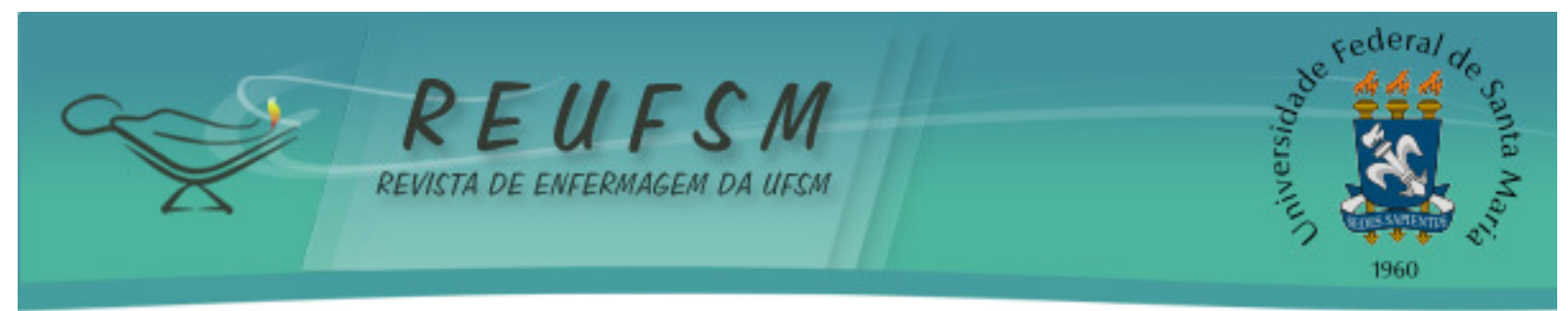

Missões/RS. A coleta de dados se deu no setor de acolhimento da ESF, no mês de novembro de 2013. Foram excluídos do estudo menores de 18 anos e sujeitos que não concordaram em participar da pesquisa.

Os dados obtidos pelo questionário estruturado foram aplicados e analisados no software Framingham General Cardiovascular Disease (10-year risk) sendo este um instrumento disponibilizado gratuitamente no site oficial do Framinghan Hearth Study. 0 instrumento analisa as variáveis: idade, sexo, diabetes mellitus, tabagismo, índice de massa corporal, pressão arterial e tratamento medicamentoso para HAS, possibilitando quantificar o risco que o usuário tem em desenvolver as seguintes patologias em um período de 10 anos: morte coronariana, infarto do miocárdio, insuficiência coronariana, angina, acidente vascular cerebral isquêmico, acidente vascular cerebral hemorrágico, ataque isquêmico transitório, doença arterial periférica e insuficiência cardíaca. 0 resultado obtido é comparado com os valores de referencia para a idade, classificados como normal e ótimo. Além disso, o software, de acordo com os fatores de risco, gera a idade vascular do sujeito em estudo.

Esta pesquisa foi aprovada pelo Comitê de Ética e Pesquisa (CEP) da Universidade Federal de Santa Maria, com parecer 437.040 em 23 de outubro de 2013, respeitando as diretrizes e normas recomendadas pela Resolução $n^{\circ} 466 / 12$ do Conselho Nacional de Saúde/MS, que regulamentam a pesquisa envolvendo seres humanos.

\section{RESULTADOS E DISCUSSÃO}

Participaram do estudo 52 sujeitos com idade entre 18 e 80 anos, compondo uma média de idade de 49 anos, destes $73,0 \%$ são do sexo feminino e $27,0 \%$ do sexo masculino. Percebeu-se uma baixa adesão de usuários do sexo masculino na amostra em estudo, esse panorama ainda é uma realidade na atenção primária do SUS, a Política Nacional de Atenção a Saúde do Homem foi lançada no ano de 2008, antes disso as ações em saúde especificas voltadas ao homem eram escassas no âmbito do SUS. O longo período sem uma política de saúde especifica e direcionada ao homem ainda repercute na baixa adesão destes usuários, especialmente na atenção primária à saúde. Também, a ausência do homem nos serviços de saúde pode ser atribuída a sua identidade social histórica, com um autocuidado inadequado, além da despreocupação com o próprio estado de saúde. ${ }^{13}$

0 índice de massa corporal (IMC) dos sujeitos investigados está descrito na figura 1. Tal variável pode ser calculada pelo quociente do peso pela altura ao quadrado, permitindo classificar os indivíduos em: baixo peso (IMC inferior a 18,5), peso normal (IMC entre 18,5 e 24,9), sobrepeso (IMC entre 25 e 29,9), obesidade Grau I (IMC entre 30 e 34,9), obesidade Grau II (IMC e 35 e 39,9) e obesidade grau III (IMC superior a 40).

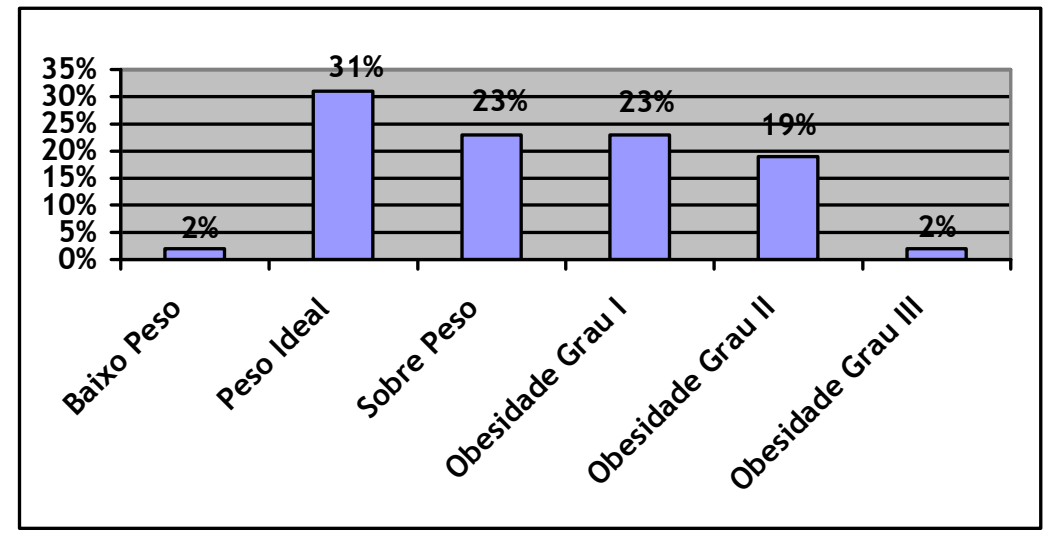

Figura 1. Índice de Massa Corporal dos sujeitos do estudo. Fonte: Dados da pesquisa, 2013. 


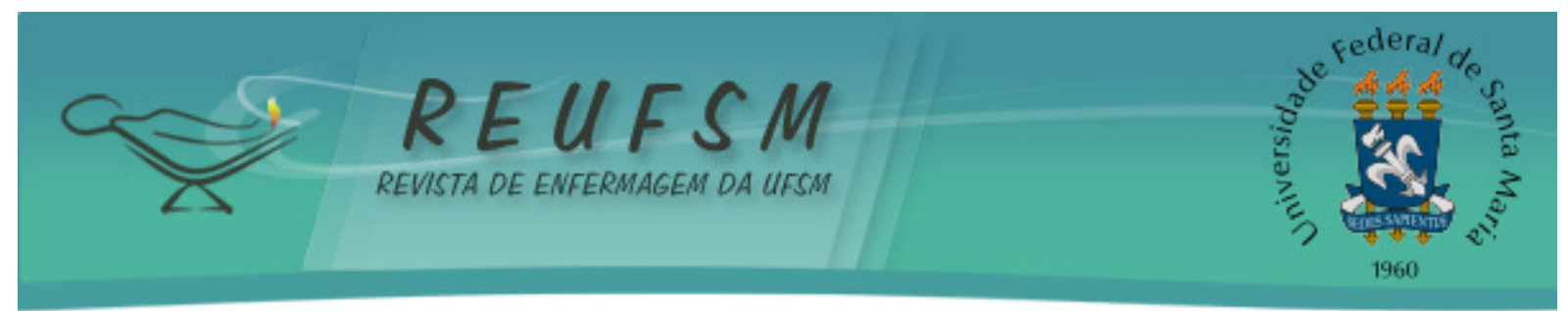

Esses dados apontaram um grande número de pessoas da amostra com o IMC elevado, pois $69,0 \%$ dos sujeitos estavam com IMC fora do ideal, sendo que $44,0 \%$ destes apresentavam-se com algum grau de obesidade. Com relação a prática de alguma atividade física foi apontado que na região sul do Brasil somente 19,3\% da população a realizam, considerando lazer e o deslocamento para o trabalho. Também, 31,1\% declararam que assistem televisão cerca de 3 horas ao dia em média. Ainda de acordo com o referido estudo, 17,4\% relataram não praticar nenhum tipo de atividade física. ${ }^{15}$

Para um bom funcionamento cardiovascular e uma boa qualidade de vida, um individuo adulto deve realizar exercícios físicos aeróbicos, no mínimo 30 minutos de forma moderada e de forma ininterrupta ou acumulada, pelo menos cinco vezes por semana. ${ }^{16} 0$ exposto acima leva a pensar que o alto grau de sedentarismo da população do sul do país pode estar relacionado com o grande número de sujeitos com IMC elevado apontado no presente estudo.

A classificação dos níveis pressóricos usada neste estudo foi referente a VI versão das Diretrizes Brasileiras de Hipertensão, da Sociedade Brasileira de Hipertensão, com base nos seguintes parâmetros: ótimo $(<120 /<80 \mathrm{mmHg})$, normal $(<130 /<85 \mathrm{mmHg})$, limítrofes $(130-130 / 85-89 \mathrm{mmHg})$, HAS grau I $(140-159 / 90-99 \mathrm{mmHg})$, HAS grau II $(160-179 / 100-$ $109 \mathrm{mmHg})$ e HAS grau III $(>180 />110 \mathrm{mmHg}) .{ }^{17}$ Os níveis pressóricos obtidos nos sujeitos pesquisados estão descritos na figura 2.

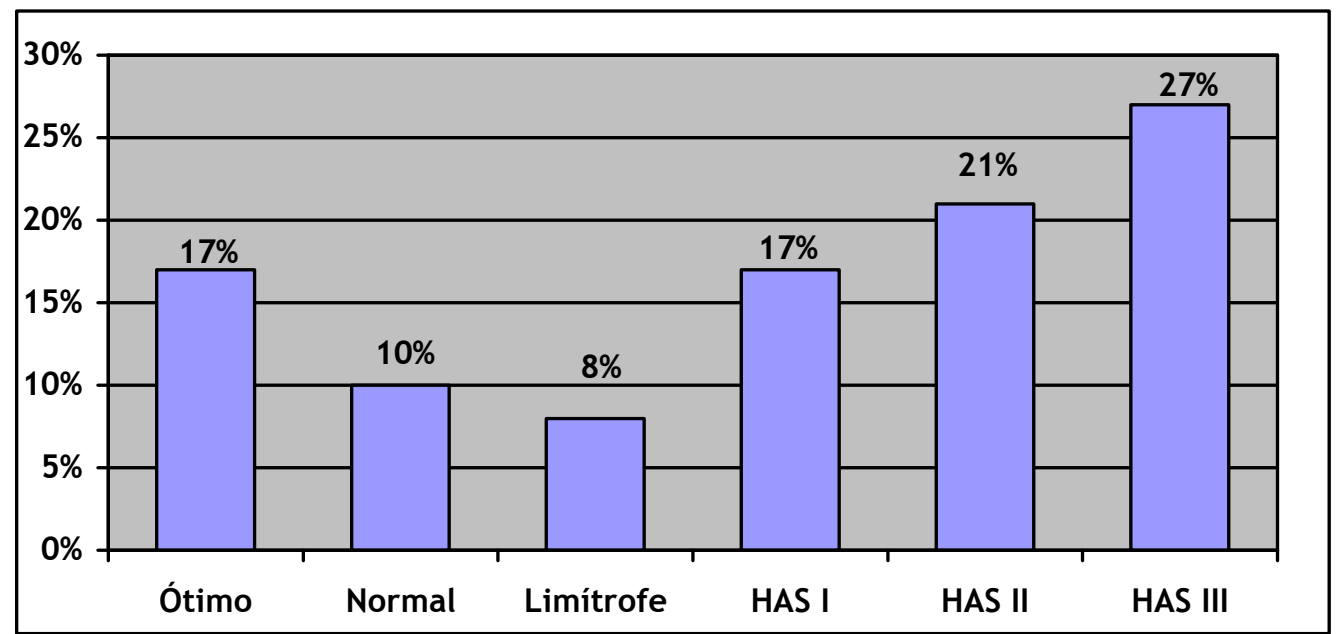

Figura 2. Distribuição dos níveis pressóricos dos sujeitos do estudo. Fonte: Dados da pesquisa, 2013.

A pesquisa evidenciou que $65,0 \%$ dos sujeitos estavam com os níveis pressóricos acima dos valores aceitáveis, destes, $21,0 \%$ relataram não fazer uso de medicação para controle dos níveis pressóricos. Além disso, $31,0 \%$ sujeitos que relataram fazer tratamento medicamentoso estavam com níveis pressóricos enquadrados em hipertensão, 13,0\% dos indivíduos que faziam uso de medicamentos para o controle da pressão arterial estavam normotensos. A HAS constitui um dos principais fatores de risco para doenças cardiovasculares e pode ocasionar lesões graves em órgãos como, coração, cérebro, rins e vasos sanguíneos. Acresce-se ainda, que a HAS é considerada um grave problema de saúde pública devido à alta demanda de custo relativo a internações, incapacitações e aposentadoria precoce. ${ }^{18}$

De acordo com pesquisa realizada que buscou comparar a qualidade de vida de sujeitos hipertensos em relação à população geral, foi evidenciado que os hipertensos possuem uma qualidade de vida menor em relação aos normotensos. Ainda de acordo com este estudo 


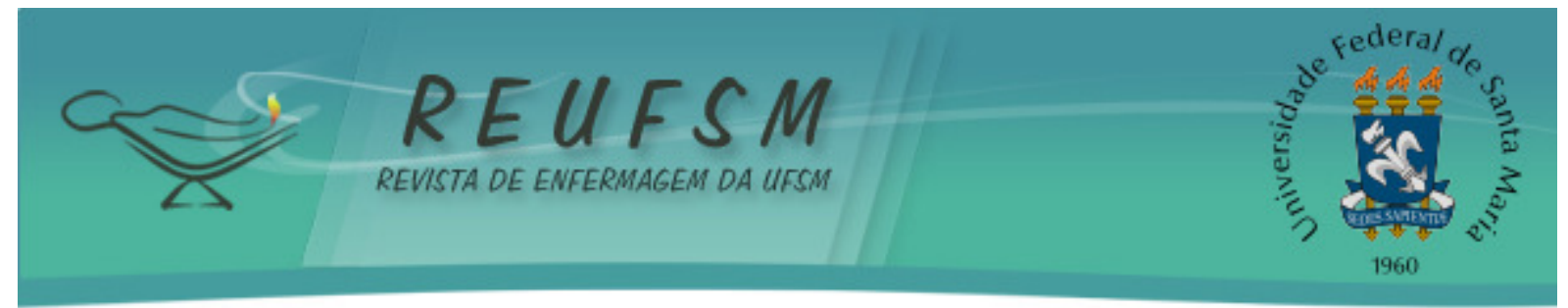

os aspectos mais impactados pela HAS na qualidade de vida foram vitalidade, capacidade funcional, dor, aspecto social, estado geral de saúde, saúde mental e aspecto físico. ${ }^{19}$

Quanto ao tabagismo, 21,0\% sujeitos relataram serem fumantes. Destes, 36,0\% são do sexo masculino e $74,0 \%$ do sexo feminino. A média de idade cronológica dos fumantes foi de 44 anos, enquanto que a média de idade vascular destes sujeitos, apontada pelo software, foi de 66 anos. Ainda a respeito dos sujeitos tabagistas do estudo, 10 deles apresentavam algum nível de HAS, e somente um sujeito encontrou-se normotenso. Em se tratando do IMC dos sujeitos que se declararam tabagistas, um encontrava-se com baixo peso, cinco com o peso ideal, um com sobrepeso e quatro com algum grau de obesidade. 0 tabagismo está diretamente relacionado como o principal fator de surgimento de diversas doenças crônicas, sendo considerado a principal causa de morte evitável em todo o mundo. ${ }^{9}$

Sendo o tabaco considerado a segunda droga mais consumida por jovens no Brasil e no mundo, acredita-se que isso é reflexo da maneira que o seu consumo é inserido na sociedade. Diversos são os fatores que contribuem para o elevado número de tabagistas, entre eles está a fácil obtenção do produto e seu baixo preço, além do grande marketing da indústria tabagista, que durante anos sustentou uma imagem positiva na sociedade ao ato de fumar. 0 sucesso destas estratégias pode ser evidenciado pelo fato de que aproximadamente $90,0 \%$ dos fumantes começam a fumar antes dos 19 anos. ${ }^{20} \mathrm{~A}$ interrupção e abandono do tabaco é uma medida fundamental e indispensável na prevenção primária e secundária das doenças cardiovasculares e de diversas outras doenças. ${ }^{21}$

A análise por meio do software possibilitou a classificação do risco para o acometimento de doenças cardiovasculares em quatro diferentes níveis: grau de risco ótimo para a idade, grau de risco normal para a idade, grau de risco elevado para a idade e grau de alto risco para idade. Os dados obtidos através do software estão descritos na figura 3.

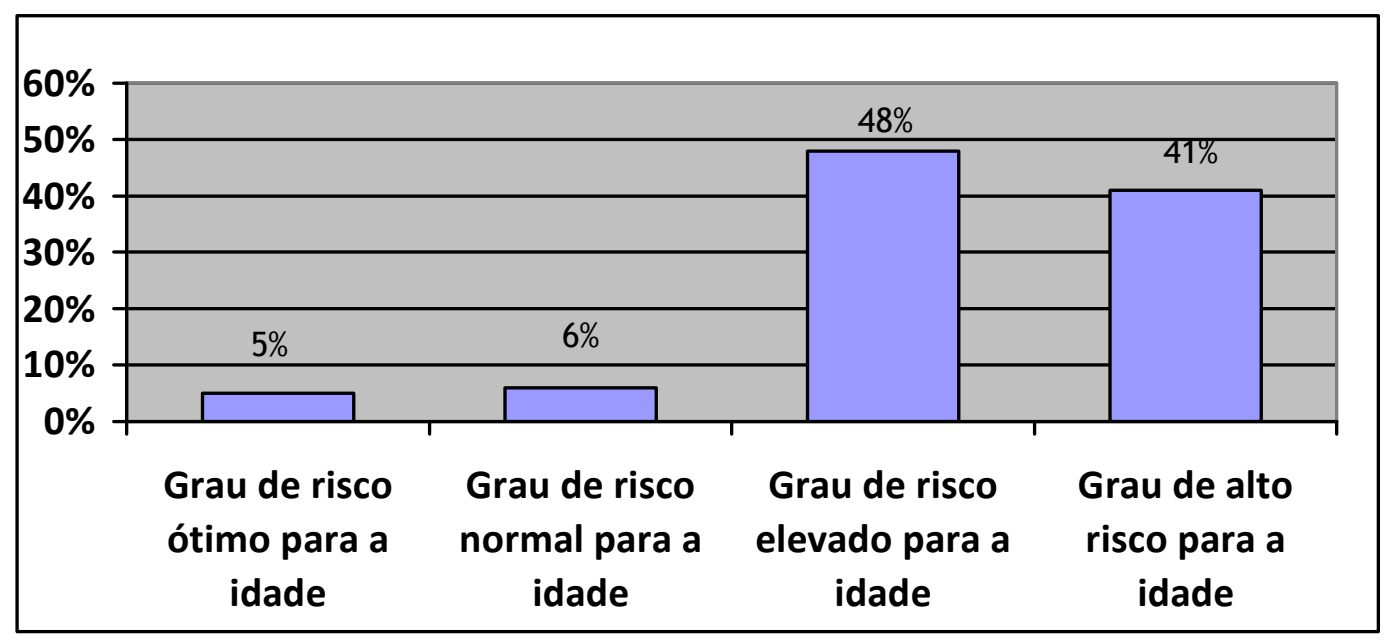

Figura 3. Grau de risco para acidentes cardiovasculares dos sujeitos do estudo. Fonte: Dados da pesquisa, 2013.

Em relação aos dados obtidos através do software, 17,0\% dos sujeitos em estudo apresentavam a idade vascular condizente ou menor que a idade cronológica. Comparando a média de idade cronológica do estudo, que foi de 49 anos, percebe-se uma notória diferença com a relação média de idade vascular, apontada pelo software, a qual foi de 61 anos. Além disso, a média de risco apontada pelo estudo foi de $19,0 \%$. Os resultados obtidos através do software evidenciam um quadro preocupante com relação ao risco para doenças cardiovasculares dos indivíduos pesquisados, pois somente $11,0 \%$ das pessoas do 


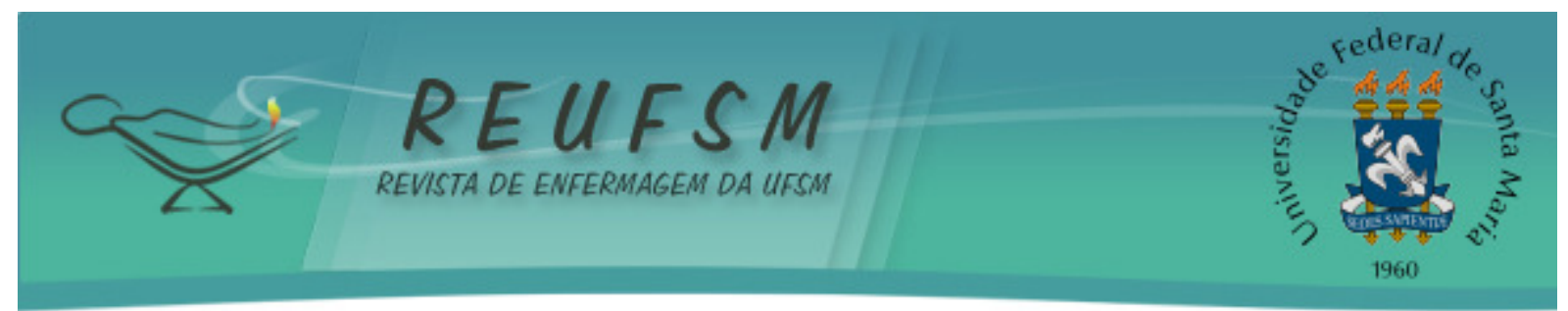

estudo encontram-se nos padrões de risco cardiovascular considerado normal ou ótimo para a idade, já grande maioria, 89,0\% estão com grau de risco acima destes valores.

Entre as pessoas com risco elevado, $41,0 \%$ apresentavam um risco superior a $20,0 \%$, classificados como indivíduos de alto risco. A análise de diversos fatores de risco simultaneamente possibilita a identificação de situações de alto risco, o que pode ser um fator motivador na adesão da proposta terapêutica, além de precipitar esforços por parte do sujeito para a sua redução. ${ }^{22}$ Com isso, a ESF configura-se como o serviço mais próximo de modificar o atual panorama das doenças cardiovasculares, pois é onde existe a possibilidade da aproximação dos profissionais com a realidade de vida dos usuários, facilitando assim as mudanças necessárias em seus comportamentos e hábitos de vida para o controle dos fatores de risco. ${ }^{23}$

A educação em saúde é um recurso de grande impacto a ser usado pelos profissionais da saúde, pois possibilita o alcance do conhecimento científico ao cotidiano dos usuários, oferecendo subsídios para adoção de novos hábitos de vida. ${ }^{24}$ Além disso, 0 Projeto Terapêutico Singular (PTS) é outra ferramenta de trabalho a ser explorada, especialmente em pessoas com grau de alto risco, pois através de um conjunto de ações terapêuticas articuladas de forma interdisciplinar onde se busca a corresposabilização do sujeito. ${ }^{25}$ Neste processo de reorganização e mudanças individuais e coletivas, o acompanhamento de usuários através da elaboração de um PTS, possui grande poder de mudanças, a medida que se incorporam planos e ações em saúde com base nos objetivos específicos de cada indivíduo.

\section{CONCLUSÃO}

As doenças cardiovasculares representam a principal causa de mortes no Brasil e com isso, demandam uma considerável parcela do orçamento anual do SUS. Ações de prevenção, pautadas na redução dos fatores de risco, são importantes para diminuir a mortalidade e o alto índice de internações hospitalares devido a estas patologias.

A quantificação de risco possibilita obter e visualizar um panorama a respeito de determinada situação e a partir disso, traçar planos de ação para intervir sobre. Evidenciou-se que boa parte dos sujeitos do estudo apresentava um elevado risco para o acometimento de doenças cardiovascular. Assim, espera-se com a realização de PTS, em indivíduos com grau de alto risco e com ações de educação em saúde no público em geral, haja redução dos fatores de risco, e, consequentemente, diminuição do risco para doenças cardiovasculares destes sujeitos.

\section{REFERÊNCIAS}

1. Brasil. Ministério da Saúde. Secretaria de Vigilância em Saúde. Secretaria de Gestão Estratégica e Participativa. Vigilância de fatores de risco e proteção para doenças crônicas por inquérito telefônico. Brasília; 2007.

2. Brasil. Ministério da Saúde. Departamento de Informática do SUS. 2013 [acesso em 2014 nov 23]. Disponível em: http: //www2.datasus.gov.br/DATASUS/index.php.

3. Monteiro CA, Moura EC, Jaime PC, Lucca A, Florindo AA, Figueiredo IRC, et al. Monitoramento de fatores de risco para doenças crônicas por entrevistas telefônicas. Rev Saúde Pública. 2005;39(1):47-57.

4. Gama GGG, Mussi FC, Pires CGS, Guimarães AC. Crenças e comportamentos de pessoas com doença arterial coronária. Ciênc Saúde Colet. 2012;17(12):3371-83. 


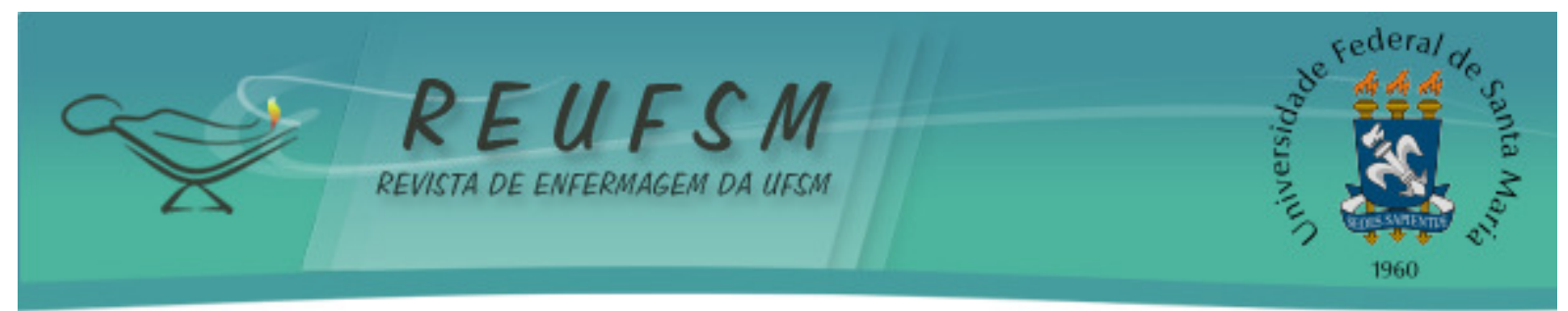

5. Woods SL, Froelicher ESS, Motzer SU. Enfermagem em cardiologia. Barueri: Manole; 2005.

6. Martins LN, Souza LS, Silva CF, Machado RS, Silva CEF, Vilagra MM, et al. Prevalência dos fatores de risco cardiovascular em adultos admitidos na unidade de dor torácica em Vassouras, RJ. Rev Bras Cardiol. 2011;24(5):299-307.

7. Viebig RF, Valero MP, Araújo F, Yamada AT, Mansur AJ. Perfil de saúde cardiovascular de uma população adulta da região metropolitana de São Paulo. Arq Bras Cardiol. 2006;86(5):621-8.

8. Rezende FAC, Rosado LEFPL, Ribeiro RCL, Vidigal FC, Vasquez ACJ, Bonard IS. Índice de massa corporal e circunferência abdominal: associação com fatores de risco cardiovascular. Arq Bras Cardiol. 2006;87(6):728-34.

9. Brasil. Ministério da Saúde. Programa Nacional de Controle do Tabagismo e outros fatores de risco de câncer [Internet]. 2003 [acesso em 2013 jun 25]. Disponível em: http://www.inca.gov.br/tabagismo/publicacoes/controle_tabagismo.pdf.

10. Mussi FC. O infarto e a ruptura com o cotidiano: possível atuação da enfermagem na prevenção. Rev Latinoam Enferm. 2004;12(5):751-9.

11. Dela Coleta MF. Modelos para pesquisa e modificação de comportamentos de saúde. Taubate: Cabral; 2004.

12. Polit DF, Beck CT, Hungler BP. Fundamentos de pesquisa em enfermagem: métodos, avaliação e utilização. Porto Alegre: Artmed; 2004.

13. Santana EN, Lima EMM, Bulhões JLF, Monteiro EMLM, Aquino JM. A atenção á saúde do homem: ações e perspectivas dos enfermeiros. REME Rev Min Enferm. 2011;15(3):324-32.

14. Knuth AG, Malta DC, Dumith SC, Pereira CA, Morais Neto OL, Temporão JG, et al. Prática de atividade física e sedentarismo em brasileiros: resultados da Pesquisa Nacional por Amostra de Domicílio (PNAD). Ciênc Saúde Colet. 2008;16(9):3697-705.

15. Sociedade Brasileira de Cardiologia, Sociedade Brasileira de Hipertensão, Sociedade Brasileira de Nefrologia. VI Diretrizes Brasileiras de Hipertensão. Arq Bras Cardiol [Internet]. 2010 [acesso em 8 maio 2015]. Disponível em: http: / / www.scielo.br/scielo.php?script=sci_arttext\&pid=S0066-

$782 \times 2010001700001 \& \operatorname{lng}=$ en.

16. Mochel EG, Almeida DS, Tobias AF, Cabral RF, Cossetti RJD. Hipertensão arterial sistêmica. Rev Hosp Univer UFMA. 2006;7(1):30-7.

17. Carvalho MV, Siqueira LB, Sousa ALL, Jardim PCBV. A influência da hipertensão arterial na qualidade de vida. Arq Bras Cardiol. 2013;100(2):164-174.

18. Pisinger C, Godtfredsen NS. Is there a health benefit of reduced tobacco consumption? A systematic review. Nicotine Tob Res. 2007;9(6):631-46.

19. Lotufo PA. O escore de risco de Framingham para doenças cardiovasculares. Rev Med São. 2008;87(4):232-7.

20. Seclen J, Fernandes As, organizadores. Experiências e desafios da atenção básica e saúde familiar: caso Brasil. Brasília: Organização Pan-Americana da Saúde; 2004.

21. Alves VSA. Um modelo de educação em saúde para o Programa Saúde da Família: pela integralidade da atenção e reorientação do modelo assistencial. Interface Comunic Saúde Educ. 2005;9(16):39-52. 


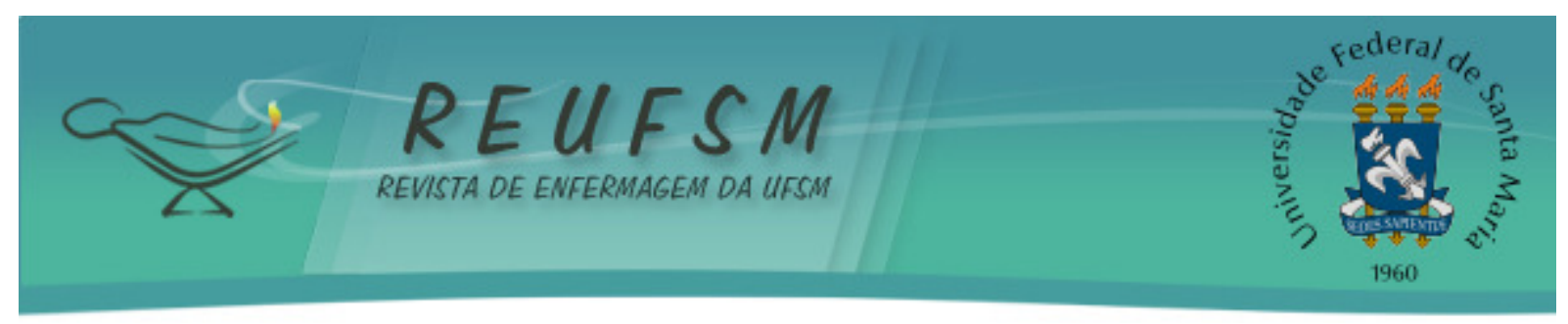

22. Brasil. Ministério da Saúde. Secretaria de Atenção à Saúde. Política Nacional de Humanização da Atenção e Gestão do SUS. Clínica ampliada e compartilhada. Brasília: Ministério da Saúde; 2009.

Data de recebimento: 03/04/2014

Data de aceite: 23/04/2015

Contato do autor responsável: Prof. Dr. Gianfábio Pimentel Franco

Endereço postal: Avenida Independência, 3751, Bairro Vista Alegre, Palmeira das Missões\RS, CEP: 98300-000, UFSM, Departamento de Enfermagem.

E-mail: gianfabiofranco@yahoo.com.br 